\title{
A rapid review of emergency department interventions for children and young people presenting with suicidal ideation
}

Farazi Virk, Julie Waine and Clio Berry

\section{Background}

Suicidal ideation is an increasingly common presentation to the paediatric emergency department. The presence of suicidal ideation is linked to acute psychiatric hospital admission and increased risk of suicide. The paediatric emergency department plays a critical role in reducing risk of suicide, strengthening protective factors and encouraging patient engagement with ongoing care.

\section{Aims}

This rapid review aims to synthesise evidence on interventions that can be implemented in the paediatric emergency department for children and adolescents presenting with suicidal ideation.

\section{Method}

Six electronic databases were searched for studies published since January 2010: PubMed, Web of Science, Medline,

PsycINFO, CINAHL and Cochrane. Outcomes of interest included suicidal ideation, engagement with out-patient services, incidence of depressive symptoms, hopelessness, family empowerment, hospital admission and feasibility of interventions. The Cochrane risk-of-bias tool was used to evaluate the quality of studies.

\section{Results}

Six studies of paediatric emergency department-initiated familybased $(n=4)$ and motivational interviewing interventions $(n=2)$ were narratively reviewed. The studies were mainly small and of varying quality. The evidence synthesis suggests that both types of intervention, when initiated by the paediatric emergency department, reduce suicidal ideation and improve patient engagement with out-patient services. Family-based interventions also showed a reduction in suicidality and improvement in family empowerment, hopelessness and depressive symptoms.

\section{Conclusions}

Paediatric emergency department-initiated interventions are crucial to reduce suicidal ideation and risk of suicide, and to enhance ongoing engagement with out-patient services. Further research is needed; however, family-based and motivational interviewing interventions could be feasibly and effectively implemented in the paediatric emergency department setting.

\section{Keywords}

Suicide; suicidal ideation; management; emergency department; psychosocial interventions.

\section{Copyright and usage}

(c) The Author(s), 2022. Published by Cambridge University Press on behalf of the Royal College of Psychiatrists. This is an Open Access article, distributed under the terms of the creative Commons Attribution licence (https://creativecommons.org/ licenses/by/4.0/), which permits unrestricted re-use, distribution, and reproduction in any medium, provided the original work is properly cited.
The paediatric emergency department (PED) plays an integral role in ensuring children and adolescents at risk of suicide have timely access to appropriate resources. Suicide rates have increased in adolescents aged 15-19 years from 3.1 to 5.7/100 000 between 2010 and 2019 in the UK. ${ }^{1}$ Approximately $13 \%$ of 5- to 19 -year-olds have at least one mental disorder; ${ }^{2,3}$ mental health presentations to a UK emergency care centre have increased threefold compared with 2019, and the most common reason for referral to Child and Adolescent Mental Health Services (CAMHS) in 13- to 17-yearolds was intentional overdose or self-harm. ${ }^{3}$ In 2018, there were 204 suicides recorded in England and Wales in young people aged 10-19 years. ${ }^{4}$ Suicide denotes the act of intentionally ending one's life. ${ }^{5}$ Mental health problems among children and young people appear to be increasing, as does suicidal ideation. Moreover, in early 2020, the COVID-19 pandemic began to place an additional significant burden on child mental health and have a substantial impact on psychosocial development. ${ }^{6}$ In Ireland, mental health attendances to the PED initially decreased by $26.8 \%$ during the first 4 months of the pandemic; by July and August, mental health presentations increased by $54.4 \%$ and $45.5 \%$ from September to December compared with 2019 data, highlighting the impact of COVID-19 on child mental health. ${ }^{7}$ Although the strongest predictor for suicide remains a previous suicide attempt, a third of adolescents who experience suicidal ideation for the first time go on to attempt suicide. ${ }^{8,9}$ Consequently, it is imperative to ensure that interventions offered to children and young people presenting to the PED are beneficial. Furthermore, the risk of a repeated suicide attempt is the highest during the first 6 months after a suicide attempt, which emphasises the importance of providing interventions that have a long-lasting effect, and of the need for robust follow-up post-discharge from the PED. ${ }^{10,11}$

A presentation of suicidal ideation has been considered as the most important sign of short-term suicide risk and warrants an in-depth clinical assessment. ${ }^{5}$ Studies have found that talking about suicide does not inadvertently create risk, and may lead to a reduction in distress in individuals who are experiencing suicidal thoughts. ${ }^{12}$ However, suicidal intent is difficult to measure, and a proportion of suicides occur as a result of individuals misjudging the risk. ${ }^{5}$ Children understand the concept of suicide and death as permanent by 8 years of age; ${ }^{13}$ nevertheless, clinicians must sensitively assess suicidal cognitions in children by in the context of rapport and empathy, within an open discussion centred around patient well-being. Worryingly, $25 \%$ of patients presenting to the PED who did not declare suicidal thoughts had suicidal ideation, ${ }^{14}$ and children and young people who died by suicide did not necessarily express recent suicidal ideation. ${ }^{15}$ Unrecognised suicidal ideation may be a result of insufficient time to explore patient well-being or a lack of mental health training for emergency department 
clinicians. ${ }^{14}$ The use of standardised screening tools is recommended. ${ }^{16}$ Results from a retrospective cohort study demonstrated that $53 \%$ of patients who presented to the PED with non-suicidal complaints were identified as having suicide risk when screened. ${ }^{17}$ Nonetheless, patients with an absence of suicidal ideation should not be deemed as having a lower risk of suicide. ${ }^{5}$ Self-harm is common in young people and engagement in these behaviours can be strongly linked to suicide. ${ }^{18}$ Self-harm refers to 'intentional self-injury without wanting to die', and frequently involves cutting, scratching, hitting and drug overdose. ${ }^{19}$ A UK study highlighted that $44 \%$ of deaths in individuals who presented to the hospital with non-fatal self-harm were attributed to suicide within the 10-18 years age group, over a 5 -year follow-up period. ${ }^{19}$ Thus, children and adolescents presenting to the PED with suicidal ideation or self-harm should be considered at suicide risk.

\section{Factors affecting youth suicide}

Effective suicide prevention strategies must be informed by the identification of factors that influence suicidality and youth suicide risk..$^{20,21}$ Suicide occurs as a result of a combination of genetic, biological and psychosocial factors. ${ }^{21}$ Suicide risk in adolescence is decreased in the context of support provision, family stability, a network of friends, a positive school environment and economic security. ${ }^{21}$ Common aetiologies for suicidal ideation in the PED include physical and mental health problems, family instability and violence, bullying and school failure, trauma and bereavement, and otherwise insufficient access to resources that aid in the development of coping skills. ${ }^{15,20,21}$ Mental health problems are perhaps the most closely associated risk factor with suicidality. ${ }^{22}$ A cohort study found that children presenting with a combination of irritability, depressive and anxiety-related symptoms in childhood (age 6-12 years) were two times more likely to think about suicide or attempt suicide during adolescence (age 13-17 years), compared with those presenting with only irritability or depressive symptoms. ${ }^{23}$ This emphasises the importance of identifying symptoms in clinical settings and providing appropriate social and emotional support to children. Moreover, children with autism spectrum disorder and attention-deficit hyperactivity disorder are at a greater risk of depression and suicidal behaviour as they progress to adulthood. ${ }^{24,25}$

\section{Assessing and screening youth suicide risk}

A universal screening tool has been proposed in a variety of medical settings, including the PED, primary care and school-based clinics. There are no standardised risk assessment tools used in the UK; however, the implementation of screening may be critical in reducing suicide, particularly for patients who do not disclose suicidal thoughts. The National Institute for Health and Care Excellence guidance advises clinicians to use the web-based tool 'STOP' to assess and monitor suicide risk in children. ${ }^{16} \mathrm{~A}$ study highlighted the benefits of using a suicide screening tool in the PED to help inform suicide prevention strategies. Ballard et al investigated the effectiveness of the Ask Suicide Screening Questionnaire on repeated PED visits. ${ }^{26}$ Results from the retrospective cohort study demonstrated that $53 \%$ of patients who presented to the PED with non-suicidal complaints also screened positive for suicide risk. ${ }^{27,28}$ Moreover, chronic childhood illnesses are significantly associated with depression in adulthood, and so addressing mental health presentations is important in reducing future suicide risk. ${ }^{28}$ Thus, screening tools that identify conditions such as autism spectrum disorder, attention-deficit hyperactivity disorder and chronic illnesses, may serve as an essential technique for assessing suicide risk and referral for emergency department interventions. $^{24}$
Currently, patients presenting with suicidal ideation are reviewed, followed up or referred to out-patient services, depending on clinical judgement. ${ }^{29}$ Longer-term out-patient treatments include psychological interventions such as cognitive-behavioural therapy (CBT), family-based interventions and motivational interviewing. ${ }^{30} \mathrm{CBT}$ is a goal-orientated therapy and involves collaboration between patients and psychotherapists to modify thought processes to facilitate change in mood. ${ }^{31}$ Family-based interventions focus on family dynamics and educating parents on signs of suicide, crisis planning and providing information on services. ${ }^{32}$ Motivational interviewing is centred on helping patients change their behaviours through listening and shared decision-making. ${ }^{33}$ However, implementing brief interventions in the PED, where patients are at high risk of suicide, may reduce short-term suicide risk and result in better engagement with out-patient follow-up. ${ }^{29}$ The World Health Organization recommends that brief interventions range from $5 \mathrm{~min}$ for brief advice to up to $30 \mathrm{~min}$ if including counselling. ${ }^{34}$ The Department of Health describes brief interventions as a vital approach for front-line workers to utilise with young people who may benefit from receiving information, and to aid in reducing harmful behaviours such as self-harm. ${ }^{29}$ Examples of brief interventions include informal discussions with youth, telephone services, one-to-one counselling within a youth programme and providing information in general practice or emergency department settings to reduce harm. ${ }^{29}$

\section{The current review}

Previous systematic reviews have been conducted on youth suicide prevention in a variety of settings, yet further research is necessary. ${ }^{35,36}$ The current review aimed to improve upon the 2010 review by Newton et al by providing a new, up-to-date systematic search and synthesis in line with Preferred Reporting Items for Systematic Reviews and Meta-Analyses (PRISMA) guidelines, ${ }^{35}$ and to improve upon the 2018 review by Robinson et $\mathrm{al}^{35}$ by focusing on PED-specific interventions. ${ }^{36}$ Therefore, in this review, we aimed to evaluate findings from brief interventions as well as other strategies that could be adapted within the PED and be beneficial for managing suicidal ideation presentations. This review focuses on psychological intervention because of the rarity of primary research trials of pharmacological interventions with young people, ${ }^{37}$ and reported longer-term benefits of psychological interventions, including reducing the burden of ongoing mental health disorders into adulthood and improved quality of life, as highlighted in recent evidence. ${ }^{38,39}$ In addition, in light of the COVID-19 pandemic, mental health presentations to the PED are expected to continue exponentially, therefore a new review must be conducted to guide future suicide prevention. ${ }^{6}$ This review restricted focus to randomised controlled trials (RCTs) only, as they are considered to provide the strongest test of whether an intervention has an effect. $^{40,41}$ By focusing on patients recruited from the PED, it may be possible to determine the factors associated with the success of specific interventions in this context.

This rapid review aimed to synthesise evidence on management interventions for children and adolescents presenting to the PED with suicidal ideation. Outcomes of interest included suicidal ideation, depressive symptoms, hopelessness, family empowerment, hospital admission, feasibility of the intervention and use of outpatient services and follow-up treatment to ascertain whether interventions improved suicidality.

The specific research questions were as follows: (a) what interventions have been used with children and adolescents presenting to the PED with suicidal ideation? and (b) what is the evidence for benefit of these interventions on suicidal ideation, associated mental health symptoms and engagement with out-patient services? 


\section{Method}

This rapid review ${ }^{42,43}$ was conducted in line with the PRISMA guidelines and conformed to the steps outlined in the 2009 PRISMA checklist. ${ }^{44}$ The protocol for this rapid review was preregistered with International Prospective Register of Systematic Reviews (PROSPERO) on 3 February 2021 (reference number CRD42021225364). Ethical approval was not required because of the retrospective nature of the study.

\section{Search strategy}

Six databases were searched on 17 December 2020: PubMed, Web of Science, Medline, PsycINFO, CINAHL and Cochrane. Other studies within the bibliography section of included studies were not searched. The following medical subject headings were used to screen titles, abstracts and keywords: 'suicidal ideation', 'emergency department', 'children', 'adolescents' and 'management'. Search terms were combined using the Boolean operators 'AND' and 'OR'. The search was restricted to articles published after January 2010. Filters including free full-text, publication date in the past 10 years and published in the English language were applied to the search results, and the full search is outlined in the Supplementary Material available at https://doi.org/10.1192/bjo. 2022.21.

\section{Selection process}

Articles were sought that reported an evaluation of any psychological/psychosocial/non-pharmacological intervention used with children or young people in the PED setting. Full inclusion and exclusion criteria are provided in Table 1.

Database search results were exported into the Mendeley software for screening (Mendeley Version 1.19.8 for Mac, Elsevier, Amsterdam, Netherlands; see https://www.mendeley.com/download-reference-manager/macOS) with the inclusion and exclusion criteria. The first author screened all records at title/abstract stage and full-text stage. The second author reviewed all full-text articles independently to determine the articles for final inclusion in the review.

\section{Data extraction}

Data were extracted by the first author using a customised Microsoft Excel version 16.43 spreadsheet. The following data were extracted: study details, design, methods, participants, intervention and outcomes, including statistical significance. Study investigators were contacted for further information, clarification or missing information as necessary.

\section{Analysis}

Because of the limited number of included studies and significant heterogenicity between outcome measures and intervention content, a full meta-analysis or sensitivity analysis was not appropriate. Therefore, studies were grouped by intervention and a range of outcome measures were analysed through narrative synthesis, ${ }^{43,45}$ using synthesis without meta-analysis guidelines. ${ }^{46}$

\section{Quality assessment}

The Cochrane Risk-of-Bias Checklist (CRBT) for RCTs was used to evaluate the quality of the included studies. ${ }^{47}$ The studies were classified into 'low risk of bias', 'high risk of bias' or 'unclear risk of bias', using an algorithm generated by the CRBT tool that highlights features of the trial that are at risk of bias. The second author assessed the quality of studies independently.

\section{Results}

\section{Study selection}

The initial literature search yielded a total of 948 articles: 33 articles were published in PubMed, 100 in Web of Science, 153 in Medline, 569 in PsycINFO, 77 in CINAHL and 16 in Cochrane (see the PRISMA flow chart in Fig. 1). After duplicates were removed, 856 articles were screened at the title/abstract stage. At full-text stage, 17 articles were screened by the first and second author. A total of six articles met the criteria for final inclusion in the review.

\section{Study characteristics}

The study characteristics for the six included studies ${ }^{48-53}$ are outlined in Table 2. All studies were published between 2010 and 2019. All included studies were conducted and published in the USA. Study sample sizes varied across all studies; the largest sample included 181 participants and the smallest sample included 49 participants. Participants were aged between 10 and 19 years. All studies took place in clinical settings ${ }^{48,50-54}$ recruited all participants in the PED setting. Participants were excluded from studies if they had signs of active psychosis, were requiring psychiatric hospital admission or had been recently discharged from hospital.

The studies evaluated family-based intervention $(n=4)$ and motivational interviewing $(n=2)$. Three family-based intervention studies conducted a brief intervention in the PED, followed by longer-term sessions post-discharge as therapy. One family-based intervention study conducted all stages of the RCT in the Department of Psychiatry at the Children's Hospital in Philadelphia. The motivational interviewing studies took place as brief interventions in the PED, including follow-up telephone calls post-discharge. Control conditions in the studies included provider education, a brief mental health referral, facilitated referrals, crisis cards and ongoing monitoring. Outcome measures differed between studies, and measures included a short-term risk of suicidal behaviour, motivation to seek follow-up treatment, suicidal ideation, depressive symptoms, family empowerment, hospital admission and feasibility of interventions. Study follow-up durations varied between 2, 3 and 6 months. Hughes and Asarnow ${ }^{50}$ did not comment on the study source of funding, but all other included studies were funded via health research grants.

\section{Study quality}

The CRBT tool was used to assess the quality of included studies. Fig. 2 summarises the risk of bias assessments. ${ }^{47}$ Two studies were assessed as a low risk of bias. ${ }^{47,48}$ Two studies were assessed as unclear risk of bias because of the lack of information regarding randomisation, allocation concealment, blinding of outcome assessors and incomplete outcome data. ${ }^{48,51}$ Two studies were given a high risk of bias; one study had missing data without explanation. ${ }^{44,45}$

\section{Type of intervention}

\section{Family-based interventions}

Four studies investigated the impact of family-based interventions on suicidal adolescents. The nature, content, duration, outcomes and follow-up period were variable across these four studies. ${ }^{48-}$ 50,53 Two studies explored the Family Intervention for Suicide Prevention (FISP) emergency department intervention, which included telephone contact post-discharge to motivate participants to engage with out-patient services. ${ }^{50,53}$ The FISP intervention by Asarnow et al involved a brief youth and family session in the PED focusing on educating families and developing a safety plan for future crises, delivered by clinicians with graduate mental 
Population Children and adolescents aged 6-19 years

At least 25\% patients recruited from the paediatric emergency department

Intervention Psychological/psychosocial/non-pharmacological interventions targeting suicidality

Comparator Any comparator, including treatment as usual

Outcomes Suicidal ideation, depressive symptoms, hopelessness, family empowerment and/or hospital admission

And/or the feasibility of the intervention

And/or out-patient services and follow-up treatment

Study design Randomised controlled trials

$\begin{array}{ll} & \text { Full text in the English language } \\ \text { Setting } & \text { Intervention deployed in clinical setting }\end{array}$

Pharmacological interventions Any country

Non-randomised controlled trials Non-English language

Published before January 2010

Interventions deployed outside clinical settings

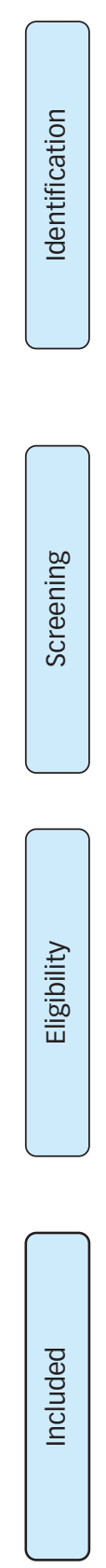

$$
\begin{gathered}
\text { PubMed: }(n=33) \\
\text { Web of Science: }(n=100) \\
\text { Medline: }(n=153) \\
\text { PsycINFO: }(n=569) \\
\text { CINAHL: }(n=77) \\
\text { Cochrane: }(n=16)
\end{gathered}
$$

Records identified through database searching:

Total studies: $(n=948)$

Records after duplicates removed

$(n=856)$

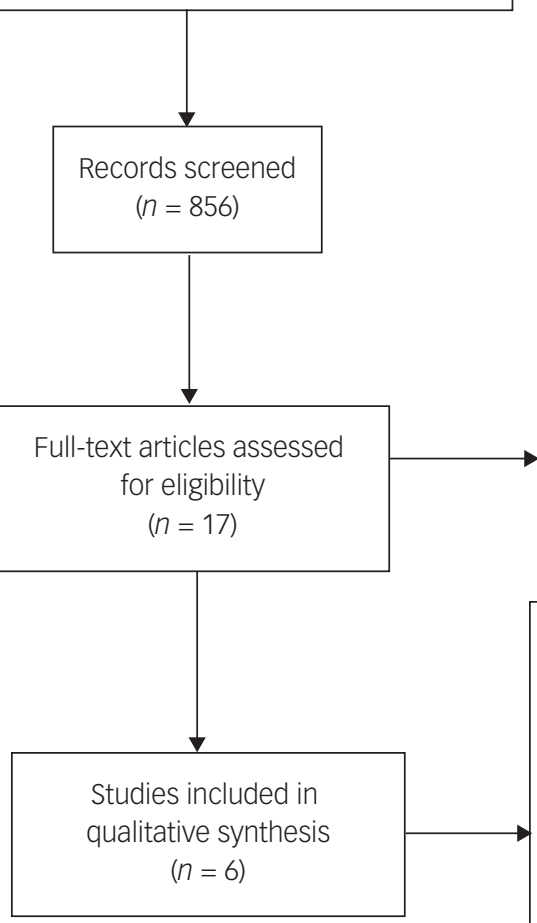

Records excluded $(n=839)$

Full-text articles excluded, with reasons:

Non-paediatric population $(n=6)$ Non-eligible setting $(n=1)$ Protocol for a study $(n=1)$ Incorrect study design $(n=1)$ Participants not recruited with suicidal ideation $(n=2)$ 
Authors (year), country

Target population

Asarnow et al (2011), Califo

enter text.

on: 'Presenting with suicide attempt and/or suicidal ideation.'

Exclusion: 'Acute psychosis/

symptoms impeding

consent/assessment; no

parent/guardian to consent

youth non English speaking

parents/guardians

non English or Spanish

speaking.'

Recruited from: PED

Diamond et al (2010),

Philadelphia, USA ${ }^{49}$ Click or

inclusion: 'Adolescents who

scored $>31$ on the SIQ and

above 20 on the BDI-II.

Exclusion: 'Adolescents needing

psychiatric hospitalisation,

recently discharged from a

psychiatric hospital, current

psychosis or mental

retardation or history of

borderline intellectual

functioning.'

Recruited from: Primary care

(75\%) and PED (25\%)

\section{$\mathrm{RCT}$}

181, age

Intervention(s)

FISP in emergency department

range designed to increase motivation for

supplemented by telephone

years, contacts after discharge.

treatment Delivered by: FISP clinicians. Clinicians

group with graduate mental health training

$n=89$, control received didactic training until

certified proficient.

$$
\text { certified proficient. }
$$

Sample $N=66$,
age range 12

ABFT: strengthening parent-adolescent EUC: a facilitated age range $12-$

bonds. Therapy starts by discussing 17 years, es adolescents to turn to his/her parent(s) when

group

$n=35$, contro group $n=31$

contemplating suicide. Followed by a session for the adolescent to identify core family conflicts linked to suicide and prepares the adolescent to speak to his or her parent(s) in the next sessions. The next task focused on parental love, empathy and parenting skills. After this families came together to discuss identified problems and practice

communication skills. The final task promoted adolescent autonomy while maintaining a family connection

Delivered by: Not reported referral process

Outcomes post-

Control condition intervention

Outcomes at follow-up

training session one Out-patient mental out treatment: only reported FISP patients post-intervention

were significantly suicidality: at follow-up, nine more likely than youths had attempted

controls to be suicide (6\%), four received

linked to out-

patient treatment

( $92 \%$ V. $76 \%$; odd

ratio $6.2 ; 95 \% \mathrm{Cl}$

1.8-21.3;

$P=0.004)$

Suicidality: only

reported at

follow-up

Depression: Not

eported post-

intervention

Suicidal ideation: post-intervention

monitoring. Other Depressive

providers set up symptoms:

initial appointments Not reported at

participant

attendance

post-intervention

suicide $(6 \%)$, four received

and five received enhanced

usual emergency care $(6 \%)$

One completed suicide

Depression: statistically

significant improvements

from baseline to follow-up:

CES-D total score $(t=-8.5$

d f $=130, P<0.0001)$, severe

CES-D (odds ratio $0.24,95 \%$

Cl $0.14-0.41, P<0.0001)$

Suicidal ideation: 24 weeks, 82.1\% of ABFT participants and $46.2 \%$ of EUC

participants reported no

suicidal ideation in the past

week (odds ratio 5.37, 95\%

1.56-18.49, $\chi 2(1)=7.66$

$P=0.006$ )

Depressive symptoms: 2

weeks follow-up, $58.1 \%$ of

ABFT participants and $38.5 \%$

of EUC participants reported

non-clinical depression

scores (odds ratio 2.21, 95\%

Cl 0.76-6.42, $\chi 2(1)=2.17$,

$P=0.14)$ utcome measure overall result and follow-up

Primary outcome:

linking patients

to out-patient

mental health

treatment and

suicidality

Exploratory

(a)

overall: effective in

linking youth to

follow-up care

and no

statistically

significant effect

on suicidality

Follow-up: 2 months

Primary outcomes: suicidal ideation and depressive symptoms

showed a slightly higher rate of mprovement for suicidal ideation. The intervention group showed significant improvements in depressive

symptoms. The number of cases of suicidal

ideation and

repetition of selfharm was similar for both groups at the postintervention

period (Continued) 


\begin{tabular}{|c|c|c|c|c|c|c|c|c|}
\hline Authors (year), country & Target population & Desigr & Participants & Intervention(s) & Control condition & $\begin{array}{l}\text { Outcomes post- } \\
\text { intervention }\end{array}$ & Outcomes at follow-up & $\begin{array}{l}\text { Outcome measure, } \\
\text { overall result and } \\
\text { follow-up }\end{array}$ \\
\hline $\begin{array}{l}\text { Grupp-Phelan et al (2019), Ohio, } \\
\text { USA } A^{52} \text { Click or tap here to } \\
\text { enter text. }\end{array}$ & $\begin{array}{l}\text { Inclusion: 'Adolescents aged } \\
\text { 12-17, positive screen for } \\
\text { suicide risk on the ASQ tool, } \\
\text { lived within } 100 \text { miles of the } \\
\text { hospital/ had no contact } \\
\text { with a mental health } \\
\text { practitioner in the } 90 \text { days } \\
\text { preceding emergency } \\
\text { department visit and stable } \\
\text { as determined by vital signs } \\
\text { and triage criteria.' } \\
\text { Exclusion: 'Chief concern of } \\
\text { suicidal behaviour/rrimary } \\
\text { or secondary psychiatric } \\
\text { concern/altered mental } \\
\text { status attributable to illness } \\
\text { or medication, lacked } \\
\text { telephone access, were } \\
\text { unable to understand the } \\
\text { study process or unable to } \\
\text { speak/read English } \\
\text { adequately to participate in } \\
\text { studyd procedures.' } \\
\text { Recruited from: PED }\end{array}$ & RCT & $\begin{array}{l}\text { Sample } \\
\quad N=168, \\
\text { age range } \\
12-17 \\
\text { years, } \\
\text { treatment } \\
\text { group } \\
n=84, \text { control } \\
\text { group } \\
n=84\end{array}$ & $\begin{array}{l}\text { Brief motivational interviewing to target } \\
\text { mental healthcare-seeking } \\
\text { behaviviour, barrier reduction } \\
\text { discussion and referral. Participants } \\
\text { received } 1 / 2.5 \text { follow-up telephone } \\
\text { calls from the social worker who } \\
\text { talked to the parent and assisted if } \\
\text { problems arose with scheduling or } \\
\text { accessing mental health treatment. } \\
\text { Telephone contact was made within } \\
2 \text { days of discharge and before the } \\
\text { scheduled appointment } \\
\text { Delivered by: social workers who } \\
\text { received a } 2 \text {-day training by a } \\
\text { Master's's level certified motivational } \\
\text { interviewing network trainer }\end{array}$ & $\begin{array}{l}\text { EUC: brief mental } \\
\text { health evaluation } \\
\text { and referral } \\
\text { following standard- } \\
\text { of-care guidelines } \\
\text { for emergency } \\
\text { behavioural health } \\
\text { assessments in } \\
\text { emergency } \\
\text { departments. } \\
\text { social workers did not } \\
\text { receive new } \\
\text { training. If the } \\
\text { adolescent was } \\
\text { safe to be } \\
\text { discharged, a } \\
\text { referral was made } \\
\text { to a mental } \\
\text { healthcare } \\
\text { practitioner during } \\
\text { a visit or the next } \\
\text { day }\end{array}$ & 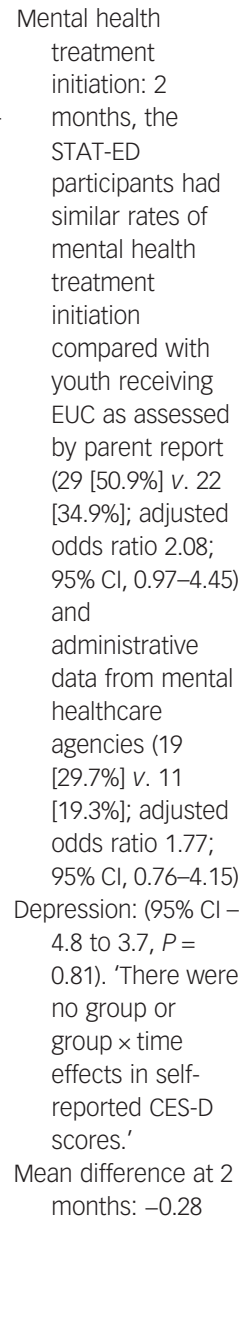 & 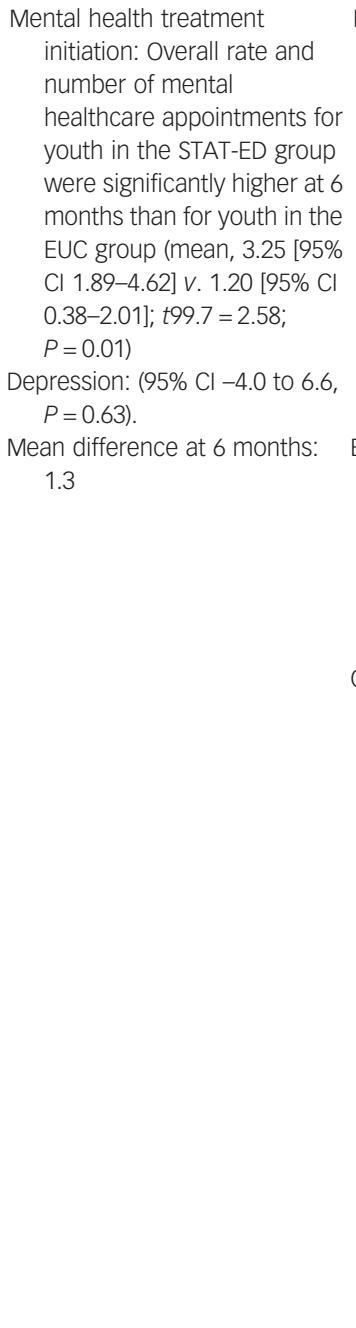 & $\begin{array}{l}\text { Primary outcomes: } \\
\text { mental health } \\
\text { treatment } \\
\text { initiation and } \\
\text { attendance } \\
6 \text { within } 2 \text { months } \\
\text { e f emergency } \\
\% \text { department } \\
\text { discharge. } \\
\text { Suicidal ideation } \\
\text { and depression } \\
\text { symptoms at } 2 \\
\text { and } 6 \text { months. } \\
\text { Exploratory } \\
\text { outcomes: } \\
\text { treatment } \\
\text { initiation and } \\
\text { attendance and } \\
\text { suicide attempts } \\
\text { at } 6 \text { months. } \\
\text { Overall: no } \\
\text { significant } \\
\text { benefit on } \\
\text { treatment } \\
\text { initiation, } \\
\text { attendance at } 2 \\
\text { months and } \\
\text { mental health } \\
\text { outcomes at } 2 \\
\text { and } 6 \text { months. In } \\
\text { exploratory } \\
\text { outcomes, STAT- } \\
\text { ED outperformed } \\
\text { EUC at } 6 \text { months } \\
\text { in linking youth } \\
\text { screening } \\
\text { positive for } \\
\text { suicide risk to } \\
\text { initial and } \\
\text { ongoing mental }\end{array}$ \\
\hline
\end{tabular}


Hughes and Asarnow (2013), California, USA ${ }^{50}$ Click or tap here to enter text.

Inclusion: 'Presenting to emergency department with

a suicide attempt and/or

Exclusion: 'No parent/guardian present to consent: acute psychotic symptoms or

symptoms that would impair

the ability to consent

complete assessments, non-

English speaking youths,

non-English or non-Spanish

speaking parents.'

King at al (2015), Michigan, USA ${ }^{51}$ Click or tap here to enter text.

speted from: PED

suicide risk screen, defined
Inclusion: 'Being 14-19 years of RC age; having a positive as suicidal ideation, recent suicide attempt or positive screens for depression and alcohol or drug abuse.

Presenting with a nonpsychiatric chief complaint.

Exclusion: 'Level one trauma (critically ill/medically unstable), significant cognitive impairment (unable to complete selfreport screen) or disposition of psychiatric

hospitalisation.'

Recruited from: PED
Sample $N=$ FISP delivered in the emergency

181, age department. A care linkage

range 10- component with follow-up

18 years, telephone contact to motivate and

treatment support linkage to out-patient

group treatment.

$n=89$, control Telephone contact: call within the first

group $\quad 48 \mathrm{~h}$ after emergency department or

$n=92$

hospital discharge with additional

contact at 1, 2 and 4 weeks

Delivered by: FISP clinicians

Sample $N=$ TOC: a crisis card with phone numbers EUC: a crisis

49, age for suicidal emergency support and

ange 14- written information. Personalised

19 years, feedback about their screening

treatment responses. Participation in an

group adapted motivational interview

$n=27$, control $\quad(35-45 \mathrm{~min}$ ) with a mental health

$n=22$

professional. Adolescents received a

follow-up note from their therapist

$2-5$ days after their visit, to support

and facilitate the implementation of their plan

Delivered by: study therapists

completed $40 \mathrm{~h}$ of training

conducted by member of the

motivational interviewing network
Usual emergency department care

enhanced by

provider education

Suicidal ideation: the Suicidal ideation: $(95 \% \mathrm{Cl}-3.3$ to Primary outcomes: values for suicidal $\quad 8.3, P=0.40$ ). A significant ideation were decrease in suicidal ideation across the groups $(f=12.42$. 3.7, $P=0.90$ ). $\quad P \leq 0.001)$

Mean difference at Mean difference at 6 months: 2 months: $-0.24 \quad 2.49$

Feasibility: Not

$$
2.49
$$

reported at pos

treatment

Suicidal ideation: Not Suicidal ideation: adolescents
reported at post- showed a decrease in $\begin{array}{ll}\text { reported at post- } & \text { showed a decrease in } \\ \text { intervention } & \text { suicidal ideation over the }\end{array}$ intervention numbers for $\begin{array}{ll}\text { suicidal emergency Depression: Not } & \text { course of the study. }(d=\end{array}$ support and written information. intervention 0.01)

opelessness: Not TOC intervention showed large reported at postpositive effects for depression $(d=1.07)$ and moderate positive effects for hopelessness at follow-up. $(d=0.40, F=9.89, P=0.01)$

Primary outcomes: suicidal ideation, hopelessness, substance use Overall: TOC

intervention greater reduction greater reduction in depressive had a nonsignificant effect on suicidal ideation, both groups showed a significant

reduction in suicidal ideation over the 2 months months

Follow-up: 2 months (Continued) 
Authors (year), country

Wharff et al (2017), Boston, USA ${ }^{48}$ Click or tap here to enter text. inclusion: 'Adolescents

escents

presenting to the emergency

department with suicidality

Adolescents considered
suicidal if, in the prior $72 \mathrm{~h}$,

suicidal if, in the prior 72
they self-identified as

suicidal, a parent/

responsible adult noted

behaviours indicating

suicidality or the adolescent

made a suicide attempt.

Presence of a consenting

presence of a consenting
parent or legal guardian with

parent or legal guardian with

whom consent resides.
Exclusion: 'Either adolescent

parent lacked fluency in

English, adolescent not

medically stable including

intoxication, adolescent

demonstrated cognitive

limitation prohibiting

completion of research

instruments, active

psychosis, physical or

medication restraint in the

emergency department.

Recruited from: PED

\section{Participants Intervention(s)}

$\mathrm{FBCl}$ received standard psychiatric

$N=142, \quad$ evaluation and experimental

age range intervention. A 60-90 min session

13-18 helping to create a joint crisis

years, narrative and taught cognitive

treatment behavioural skill-building

group therapeutic readiness,

$n=71, \quad$ psychoeducation about depression

control and safety planning. The clinical

group

team made recommendations for

treatment with input from the patient and family.

Delivered by: psychiatric social workers who were trained in the intervention whore psychiatric

evaluation and

clinic/discharge

EUC: social workers
did not receive new
training. If the
adolescent was
safe to be
discharged, a
referral was made
to a mental
healthcare
practitioner during
a visit or the next
day.
TAU included standar
psychiatric
evaluation and
clinic/discharge
recommendations

Outcomes post-

intervention

suicidality: no

statistically

significant

change in RFL-A

post-

empowerment:

$\mathrm{FBCl}$ showed

higher scores for

FES compared

with $\operatorname{TAU}\left(F_{1,121}=\right.$

$8.1, P<0.01)$

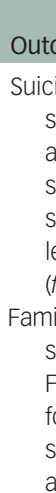

Outcomes at follow-up

icidality: no statistically

significant change in RFL-A

scores increased over the

study period indicating lower

levels of suicidality.

$(f=23.1, P<0.001)$

empowerment: $\mathrm{FBCl}$

showed higher scores for

follow-up. Parents reported

statistically increases in FES

at 1-month follow-up.

$\left(F_{4,431}=23.0, P=0.005\right)$

Hospital admission: FBCI

participants were less likely

to be admitted to hospital

compared with TAU (odds

ratio, 3.4; 95\% Cl 1.7-6.8;

$P<0.005)$
Outcome measure

overall result and

(a)

presence and

severity of

adolescent

suicidality, family empowerment,

post-emergency

department

recommendation

and disposition

ondary outcome: parent/guardian

satisfaction and

emergency

department

recidivism over

the 1 month after

the emergency

department visit

Overall: All

participants

reported lowe

levels of

suicidality

compared with

baseline. $\mathrm{FBCl}$

group showed

significantly

higher scores for

family

empowerment

and patient

satisfaction. FBCI

participants were

significantly less

likely to be

admitted to

hospital

Follow-up: 3 months

PED, paediatric emergency department; RCT, randomised controlled trial; FISP, Family Intervention for Suicide Prevention; EUC, enhanced usual care; CES-D, Center for Epidemiological Studies for Depression; SIQ, Suicidal Ideation Questionnaire Junior; BDI-II, Beck Depression Inventory Il; ABFT, attention-based family therapy; ASQ, Ask Suicide Screening Questions; STAT-ED, Suicidal Teens Accessing Treatment After an Emergency Department Visit; TOC, Teen Options for Change; FBCI, Family-Based Crisis intervention; TAU, treatment as usual; FFL-A,
Reasons for Living Inventory for Adolescents; FES, Family Empowerment Scale. 


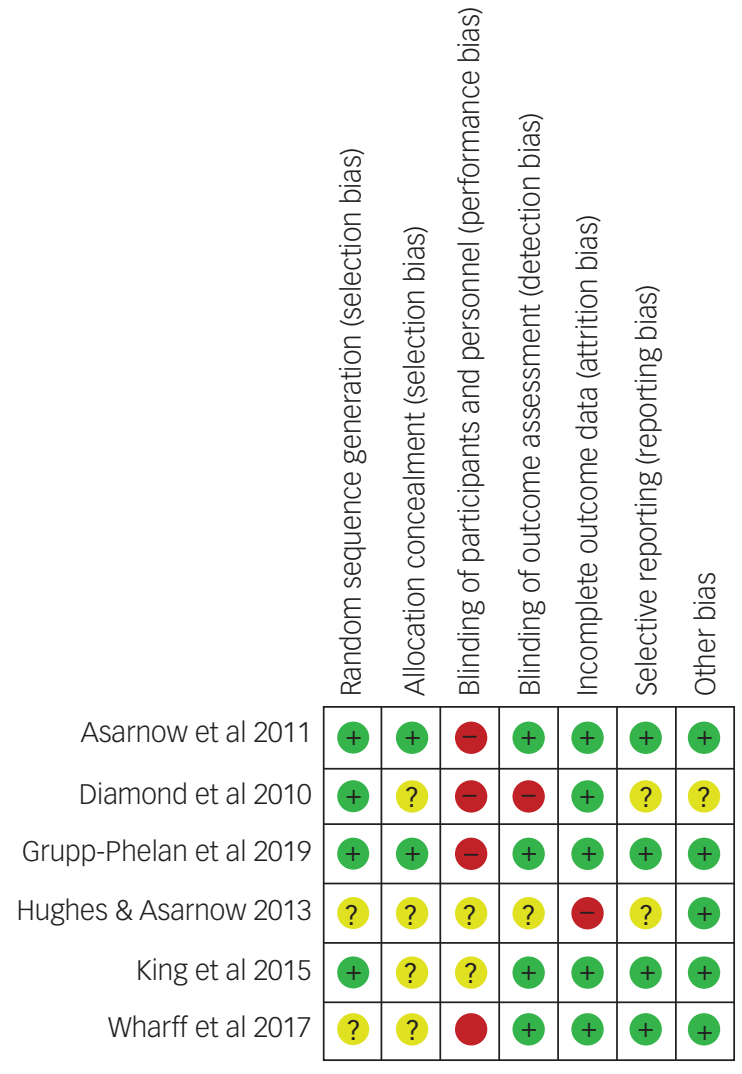

Fig. 2 Summary of the risk of bias assessment with the Cochrane Risk-of-Bias Checklist.

health training who received didactic training with role playing. ${ }^{53}$ Following this session, structured telephone contacts were made to youth to motivate and support out-patient treatment within 48 $\mathrm{h}$ of discharge. ${ }^{53}$ Additional contacts were made at 1,2 and 4 weeks post-discharge. Hughes and Asarnow designed the FISP intervention as a brief youth and family therapy session delivered in the emergency department. ${ }^{50}$ The full FISP intervention was delivered to $80.9 \%$ of participants in the emergency department; for youth discharged before completion of the FISP, the intervention was delivered on in-patient units after transfer from emergency department (12.4\%), other community locations $(3.4 \%)$ or via telephone $(2.4 \%) .{ }^{53}$ Youth were discharged with a safety plan card with coping strategies and useful contacts. ${ }^{53}$ The intervention was delivered by clinicians with graduate training in psychology, social work, psychiatry or a related mental health field. ${ }^{53}$ Youth were contacted via telephone within $48 \mathrm{~h}$ of discharge, and additional contacts were made at 1,2 and 4 weeks post-discharge. ${ }^{53}$ Diamond et al investigated an intervention, referred to as attention-based family therapy (ABFT), that focused on strengthening parent-adolescent bonds through face-to-face sessions, and was delivered by $\mathrm{PhD}$ or Master of Social Work level therapists who were trained by two of the study authors. ${ }^{49}$ Over six to eight sessions, participants completed five tasks that promoted family connectedness and adolescent autonomy. ${ }^{49}$ Parents were present for four to six sessions and adolescents completed two tasks alone. ${ }^{49}$ Wharff et al investigated the family-based crisis intervention (FBCI), designed to take place in the emergency department and delivered by trained psychiatric social workers. ${ }^{48}$ This involved psychiatric evaluation, including a 60-90 min session helping families with psychoeducation and safety planning. ${ }^{48}$ Participant follow-up durations differed between studies, ranging from 2 to 6 months. ${ }^{48-50,53}$

\section{Motivational interviewing interventions}

Two studies examined the effects of motivational interviewing on suicidal adolescents; Grupp-Phelan et al also explored the impact of motivational interviewing on treatment initiation and attendance within 2 months of discharge from the PED. ${ }^{51,52}$ The nature of motivational interviewing intervention differed between the two studies, but both interventions took place within the PED. The study by Grupp-Phelan et al involved four brief motivational interviewing sessions delivered to the adolescent and parent by trained social workers that targeted mental healthcare-seeking behaviour, barrier reduction discussion and referral. ${ }^{52}$ The adolescent and parent were interviewed alone for the first session; subsequent sessions involved the adolescent and parent together to discuss mental health options, potential barriers and next steps. ${ }^{52}$ After these sessions, participants received one or two follow-up telephone calls to discuss potential problems within their out-patient mental health treatment. ${ }^{52}$ Participants were followed up at 2 and 6 months. ${ }^{52}$ In the study by King et al, participants received a 35-40 min motivational interviewing session with a certified motivational interviewing mental health professional and a handwritten note from their therapist 2-5 days post-discharge; follow-up took place over 2 months. ${ }^{51}$ The study team also gave participants a crisis card for emergency suicidal support contacts and written information regarding depression, suicide risk, firearm safety and local mental health services. ${ }^{51}$

\section{Outcomes of interventions}

\section{Suicidal ideation}

Five studies examined the impact of interventions on suicidal ideation, and outcome measures varied across studies. ${ }^{48,49,51-53}$ One study measured suicidal ideation with the Harkavy-Asnis Suicide Scale, to assess active and passive suicidal ideation. ${ }^{53}$ Three studies used the Suicidal Ideation Questionnaire-Junior to assess suicidal ideation. ${ }^{48,51,52}$ One study measured change over time in adolescent suicidality (Reasons for Living Inventory for Adolescents; RFL-A). ${ }^{48}$ Asarnow et al evaluated suicidality as an exploratory outcome; results illustrated no statistically significant intervention effects on suicidality. ${ }^{53}$ In the motivational interviewing intervention by Grupp-Phelan et al, there was a significant decrease in suicidal ideation across groups. ${ }^{52}$ Diamond et al found a slightly higher rate of improvement owing to a rapid reduction in suicidal ideation in the ABFT intervention group compared with the control group. ${ }^{49}$ At the end of the follow-up period, $82.1 \%$ of participants receiving the intervention reported no suicidal ideation in the past week compared with $46.2 \%$ of enhanced usual care (EUC) participants. ${ }^{49}$ Over the 6-month follow-up period, four out of 35 intervention group participants (11.4\%) had made a suicide attempt, compared with seven out of 33 (21.2\%) EUC participants. ${ }^{49}$ King et al reported a significant decrease in time for suicidal ideation over the study period. ${ }^{51}$ Wharff et al reported increases in the mean RFL-A total scores over the study period; however, there were no significant differences between the groups. ${ }^{48}$ This intervention illustrated that participants had lower levels of suicidality over time at 1-month follow-up compared with their baseline assessment. ${ }^{48}$

\section{Depressive symptoms and hopelessness}

Three studies explored the impact of the intervention on depressive symptoms. ${ }^{49,51,52}$ Diamond et al measured depression with the selfreport Beck Depression Inventory, and results showed significant effects supported by large effect sizes. ${ }^{49}$ After treatment, at 6month follow-up, $54.8 \%$ of ABFT participants and $31.0 \%$ of EUC participants had non-clinical depression scores. ${ }^{49}$ The Reynold 
Adolescent Depression Scale Short Form was used by King et al to measure depression; intervention (Teen Options for Change; TOC) participants demonstrated a significant positive change in depression, with a large effect size from baseline to follow-up. ${ }^{51}$ King et al also measured hopelessness with the Beck Hopelessness Scale, and results showed a moderate effect size for hopelessness. ${ }^{51}$ In contrast, Grupp-Phelan et al results showed no significant difference in depressive symptoms between intervention (Suicidal Teens Accessing Treatment after an Emergency Department Visit; STATED) and EUC groups. ${ }^{52}$

\section{Engagement with out-patient services}

Two studies investigated the impact of interventions on engagement with out-patient services and treatment initiation. ${ }^{52,53}$ GruppPhelan et al explored treatment initiation and attendance. Exploratory outcomes showed no significant difference between the STAT-ED intervention and EUC in the rate of mental health appointments at 2 -month follow-up. ${ }^{52}$ However, by 6 months, follow-up participants in the STAT-ED group were more likely to initiate mental health treatment and the overall rate of mental health appointments were significantly higher in the STAT-ED group compared with EUC. ${ }^{52}$ Asarnow et al intervention included a telephone contact within $48 \mathrm{~h}$ of discharge from the PED, to motivate and support out-patient treatment. ${ }^{53}$ More FISP participants were likely to receive out-patient treatment and had significantly more visits compared with the control. ${ }^{53}$

\section{Family empowerment}

In one study, family empowerment was measured as an outcome. ${ }^{48}$ Scores were obtained with a 34-item self-report Family Empowerment Scale (FES) that measures the level of empowerment of parents of a child with emotional difficulties. ${ }^{48}$ The FES questionnaire is completed by parents to assesses family, child and parental involvement within the community. ${ }^{55}$ Parents answer questions such as 'I feel I am a good parent', 'I make sure I stay in regular contact with professionals who are providing my child services' and 'I have ideas about the ideal service system for children'. ${ }^{55}$ The scoring scale is rated $1-5 ; 1$ equates to 'never' and 5 to 'very often' ${ }^{55}$ Wharff et al reported higher scores for family empowerment during the study. ${ }^{48}$ At the 1 -month follow-up, there were statistically significant increases in the FES score. ${ }^{48}$

\section{Hospital admission}

One study evaluated the impact of the intervention on in-patient psychiatric hospital stay. ${ }^{48}$ The FBCI demonstrated that participants randomised to the intervention were significantly less likely to be admitted to hospital compared with treatment as usual. ${ }^{48}$ During the study, $68 \%$ of treatment-as-usual participants were admitted to hospital, compared with only $38 \%$ of $\mathrm{FBCI}$ participants. ${ }^{48}$

\section{Feasibility}

Hughes and Asarnow conducted a follow-up study of Asarnow et al to ascertain the feasibility of delivering FISP in the PED. ${ }^{50,53}$ Results showed that $80.9 \%$ received the intervention in the PED; however, because of discharge, FISP was delivered on in-patient units $(12.4 \%)$, in the community $(3.4 \%)$ or by phone $(2.2 \%) .^{50}$ In addition, $78.7 \%$ of FISP sessions were delivered with a parent and youth; however, $16.9 \%$ of FISP sessions were conducted with youth only, as some youth were brought to the PED by ambulance or police without their parents. ${ }^{50}$ Telephone calls were made to youth to enhance motivation and support for follow-up treatment at $48 \mathrm{~h}$ and 1,2 and 4 weeks post-discharge; however, $88.8 \%$ of youth received at least one telephone call. ${ }^{50}$ This highlights potential barriers that become apparent after discharge, as successful contact with families requires clinicians and families to work together effectively. In addition, three participants withdrew from FBCI in the study by Wharff et al, and ten participants were lost to follow-up owing to being unable to reach by telephone. ${ }^{48}$ Similarly, four participants receiving TOC were discharged or left the hospital before motivational interviewing took place; three participants were lost to follow-up. ${ }^{51}$

\section{Discussion}

This rapid review aimed to investigate interventions used in the PED setting for children and adolescents presenting with suicidal ideation. Six studies met the review inclusion criteria. All studies were initiated in the PED. The studies provided evidence for the impact of these interventions on suicidal ideation. ${ }^{48-53}$ Studies also outlined positive effects of interventions on patient engagement with out-patient follow-up treatment, depressive symptoms, hopelessness, family empowerment, hospital admission and intervention feasibility ${ }^{48-53}$ To our knowledge, our study is the most recent and first rapid review to focus on a broad range of outcome measures to support PED care for young people presenting with suicidal ideation, as well as to identify areas requiring further research.

Two potential interventions were identified in this review; four studies involved family-based interventions and two studies comprised motivational interviewing interventions. ${ }^{48-53}$ Overall, findings suggest that family-based interventions are associated with a reduction in suicidal ideation, whereas evidence for the benefit of motivational interviewing is more equivocal. Overall, there is a lack of high-quality evidence because several limitations within the included studies, and therefore the conclusions should be drawn with caution.

Included studies that investigated the effects of family-based interventions on suicidal ideation consisted of dedicated sessions with families and patients in the PED to strengthen family bonds during a time of crisis. This is in keeping with a clinical review that highlighted early involvement of the family, formulation of risk evaluations and care based upon suicide risk and the availability of resources promote better outcomes. ${ }^{56}$ One study measured family empowerment and found statistically significant increases in the FES score; ${ }^{48}$ thus, it may be that the impact of family interventions is through the mechanism of empowering the family and mobilising family-based coping. Nonetheless, more high-quality studies investigating family-based interventions are required, with specific attention to the mechanisms of impact. However, a focus on family-based interventions must not detract from the importance of alternative intervention options in situations where family intervention may be inappropriate or unsafe; for example, for looked after children, or in the context of family conflict or domestic violence or abuse. Thus, it is important for the PED to be equipped with multiple intervention options and the skills to negotiate appropriate intervention provision, while retaining an atmosphere of collaborative patient care.

Furthermore, family-based interventions and motivational interviewing show some effect on depressive symptoms and hopelessness. Previous studies have $e^{23}$ suggested suicidality is linked to the experience of mental health problems such as depression. ${ }^{22}$ Moreover, hopelessness is implicated in suicidality, with greater hopelessness differentiating adolescents who attempt suicide from those with suicidal ideation but no attempts. ${ }^{22}$ However, further research is necessary to evaluate whether reductions in depression and hopelessness result in a reduction in suicidality. 
An important component of suicide prevention is out-patient engagement, as studies have shown that patients who engage with services have a decreased risk of suicide. ${ }^{57}$ Two studies demonstrated that family-based interventions ${ }^{50}$ and motivational interviewing $^{52}$ can increase out-patient treatment initiation and service use within the immediate 2 days after PED discharge ${ }^{50}$ and over the longer-term, i.e. 6 months after the intervention. ${ }^{52}$ In the study evaluating motivational interviewing, efforts were made in the intervention group to follow up on patients to check whether they were able to attend scheduled appointments, and telephone calls were made within 2 days post-discharge. ${ }^{52}$ The timing of follow-up contact has been highlighted as an important factor in managing suicide risk in patients who have been discharged after psychiatric hospital stay. ${ }^{57}$ A recent cohort study ${ }^{58,59}$ found that youth who had an out-patient mental health visit within 7 days after discharge had a decreased risk of suicide during the 6 months after psychiatric hospital stay. ${ }^{57}$ Thus, as a suicide prevention effort, contact must be made with patients within 7 days of discharge from any clinical setting. ${ }^{57}$

\section{Strengths and limitations}

This novel rapid review has several strengths. First, the search of six high-yield databases facilitated a comprehensive search of relevant literature. ${ }^{44}$ Studies published within the past 10 years were included, which ensured that our conclusions were up to date. Only RCTs were eligible for this review; RCTs are considered the most valuable study methods for generating reliable high-quality data and assessing the effectiveness of interventions. ${ }^{40,41}$ The screening process was undertaken by one reviewer and the data extraction and quality assessment were checked by a second reviewer, to minimise bias during this process. Although the main outcome measure was a reduction in suicidal ideation, a broader set of outcomes were considered to ensure inclusion of additional factors associated with ongoing suicide risk and intervention implementation.

Some important limitations must nonetheless be borne in mind. Eligibility criteria were limited to studies published in the English language; broadening the criteria to non-English language studies may have resulted in additional studies, albeit their relevance to the UK healthcare system may be limited. Furthermore, this review yielded a small number of studies that displayed significant heterogeneity in interventions, outcomes and population. As such, a meta-analysis could not be performed because variations in interventions and outcomes, and primary research that has considerable risk of bias, may produce misleading or inappropriate meta-analytic results. ${ }^{46}$ Therefore, we made no pooled estimate of intervention effectiveness.

In addition, an important consideration is the exclusion of severe cases of suicidal ideation within reviewed studies; therefore, results may represent effects with young people presenting with less severe suicidality than seen in the PED generally. This reflects a broad tension in research trials around maximising the reach of an intervention (and research outcomes) to people potentially most at need of support as well as balancing safety concerns. Safety is an important consideration because psychological interventions may cause harm as well as give rise to benefits, and negative experiences of care immediately after events such as self-harm are seen to increase risk of further self-harm and hinder future disclosure. ${ }^{60}$ Current UK guidelines are to make an urgent referral to CAMHS for children and young people presenting with high risk of suicide (and depression), with the provision of a safe space to prevent injury as needed, and not to provide any psychological intervention in situ. ${ }^{61}$ Nonetheless, evidence for effective interventions that could be safely deployed in the PED for high-risk children and young people, in the context of the very high demand on CAMHS services, ${ }^{62}$ could build much-needed health service capacity and help to prevent deaths by suicide. The development of intervention protocols and evidence regarding intervention safety and effectiveness for adults and young people in the high-risk suicidality spectrum remains an important goal. ${ }^{63}$

All included studies were published and conducted in the USA; this highlights that the results of the review may not translate to the UK or other countries. ${ }^{48-53}$ Consequently, there are implications for the universal application of the interventions to other healthcare systems. For example, in the UK, mental health service funding is significantly limited; therefore, replicating the interventions in UK hospitals might be difficult. ${ }^{64} \mathrm{~A}$ literature review and thematic analysis of emergency department staff attitudes toward patients with a mental health problem highlighted that staff perceived caring for individuals with a mental health concern as a challenge and felt ill-prepared in assessing individuals. ${ }^{65}$ Therefore, this demonstrates that there is a lack of confidence in emergency department staff when approaching mental health presentations.

The studies reviewed were largely at high risk of bias. Many studies did not publish protocols or outline randomisation processes, and sample sizes were relatively small. ${ }^{48-53}$ Moreover, two studies $^{48,52}$ recruited participants within restricted staff working hours, which was reported as office hours only in one study, ${ }^{52}$ and therefore the samples may not be representative of children presenting outside of usual office hours. Diamond et al recruited $75 \%$ of participants from primary care and $25 \%$ from the PED. ${ }^{49}$ This study did not disaggregate results for primary care and PED participants; there is a possibility that participants recruited through primary care differ in terms of initial presentation and response to intervention. ${ }^{49}$ Moreover, the eligibility criteria for participants in this review ranged from 6 to 19 years; however, the age of participants ranged from 10 to 19 years within included studies. ${ }^{48-53}$ Therefore, as the included studies did not test the intervention with children aged under 10 years, we could make no conclusions about the effectiveness and feasibility of delivering interventions in the PED in this group. However, children show signs of emotional and behavioural distress when exposed to parental conflict, and so it is important that family-based interventions are appropriate for all age groups and adapted for younger children. ${ }^{66,67}$

\section{Implications and priorities for future research}

This review identified two interventions that demonstrated some improvements in suicidal ideation, with stronger evidence for the effectiveness of family-based interventions, especially regarding out-patient engagement. Currently, in the UK, patients requiring hospital admission are admitted as an in-patient; a child mental health liaison team within an acute hospital setting is rare, therefore patients are seen by a CAMHS professional the following day or they may wait several hours before seeing CAMHS within the PED. There is sufficient evidence to highlight the role of family as a protective factor against suicide; promoting cohesion and education of parents and children leads to better outcomes. ${ }^{15}$ Our results have shown promising approaches to family-based therapy, particularly ABFT. ${ }^{49}$ Based upon the literature supporting the importance of family-child relationships in suicidality onset and outcomes, we propose a family-based intervention within the PED and contact within 2 days post-discharge in a follow-up clinic. ${ }^{16,44,58}$ A priority must be to use a co-design process with children, young people, families and PED professionals to adapt interventions used in the USA for appropriate delivery in the UK PED setting. However, we acknowledge that some young people have difficult family relationships or do not have contact with parents or a guardian, such as 
looked after children, thus brief motivational interviewing may be an appropriate alternative. ${ }^{56}$ Although our review focused on managing suicidal ideation, we recommend training for emergency department staff in both being able to screen, assess and effectively identify young patients with suicidality, ${ }^{68}$ and in delivering brief psychological interventions in the PED setting. ${ }^{29}$ This would encourage patients to seek out-patient follow-up treatment, prevent readmission and keep costs minimal, which may aid in supporting community suicide prevention efforts.

This review has highlighted the lack of high-quality evidence to support the implementation of evidence-based interventions for youth suicidality in the PED setting. Thus, we recommend highquality randomised trials with larger sample sizes, investigating and comparing family intervention and motivational interviewing approaches alongside other promising interventions. We recommend studies consider relevant subpopulations, including the evaluation of alternative interventions not involving family as relevant depending on family circumstances; for example, young people at very high risk of suicide, looked after children, and children with historical and/or current experiences of domestic violence and abuse. However, involving family where appropriate, by asking family empowerment questions within the PED to ascertain how families are coping, may result in better patient outcomes. We recommend performing cost-effectiveness analyses of potential interventions, ${ }^{64}$ to ensure intervention delivery would be cost-effective and sustainable. These recommendations would enable future systematic reviews and meta-analyses to be based upon more reliable studies.

Finally, despite the significant recent rises in suicide rates in young people generally and throughout the COVID-19 pandemic, there is limited high-quality evidence to illustrate the effectiveness of interventions. This review highlights the apparent benefits of psychological interventions delivered within the PED setting for children and young people presenting with suicidality, including improving mental health, depressive symptoms, hopelessness, family empowerment and hospital admission. Therefore, it is imperative to conduct more high-quality research to clarify definitive intervention outcomes. Studies must be undertaken within the UK specifically to establish successful emergency department-based interventions that can work effectively within this context.

Farazi Virk (D), Brighton and Sussex Medical School, University of Sussex, UK; Julie Waine, Mental Health Liaison Team, Queen Alexandra Hospital, UK; Clio Berry (1D, Brighton and Sussex Medical School, University of Sussex, UK

Correspondence: Farazi Virk. Email: f.virk1@uni.bsms.ac.uk

First received 15 Sep 2021, final revision 14 Jan 2022, accepted 31 Jan 2022

\section{Supplementary material}

Supplementary material is available online at https://doi.org/10.1192/bjo.2022.21.

\section{Data availability}

The data that support the findings of this study are available from the corresponding author, F.V., upon reasonable request.

\section{Author contributions}

All authors substantially contributed to the conception and design of the work, including drafting and final approval of the version to be published.

\section{Funding}

This research received no specific grant from any funding agency, commercial or not-for-profit sectors.

\section{Declaration of interest}

None.

\section{References}

1 Office for National Statistics. Suicides in England and Wales. Office for National Statistics, 2021 (https://www.ons.gov.uk/peoplepopulationandcommu nity/birthsdeathsandmarriages/deaths/datasets/suicidesintheunitedkingdomre ferencetables).

2 The Lancet. Child mental health services in England: a continuing crisis. Lancet 2020; 395(10222): 389.

3 McCall B. Threefold Rise in Mental Health Referrals from Paediatric Emergency during COVID-19. Medscape, 2021 (https://www.medscape.com/viewarticle/ 953755).

4 Office for National Statistics. Number of Suicides by Single Year of Age, England and Wales, 2018 Registrations. Office for National Statistics, 2019 (https:// www.ons.gov.uk/peoplepopulationandcommunity/birthsdeathsandmarriages/ deaths/adhocs/10941numberofsuicidesbysingleyearofageenglandandwales 2018registrations).

5 Cash SJ, Bridge JA. Epidemiology of youth suicide and suicidal behavior. Curr Opin Pediatr 2009; 21: 613-9.

6 Singh S, Roy D, Sinha K, Parveen S, Sharma G, Joshi G. Impact of COVID-19 and lockdown on mental health of children and adolescents: a narrative review with recommendations. Psychiatry Res 2020; 293: 113429.

7 McDonnell T, Barrett M, McNicholas F, Barrett E, Conlon C, Cummins F, et al. Increased mental health presentations by children aged 5-15 at emergency departments during the first 12 months of COVID-19. Ir Med J 2021; 114(5): 356.

8 Nock MK, Borges G, Bromet EJ, Alonso J, Angermeyer M, Beautrais A, et al. Cross-national prevalence and risk factors for suicidal ideation, plans and attempts. Br J Psychiatry 2008; 192(2): 98-105.

9 Inagaki M, Kawashima Y, Yonemoto N, Yamada M. Active contact and followup interventions to prevent repeat suicide attempts during high-risk periods among patients admitted to emergency departments for suicidal behavior: a systematic review and meta-analysis. BMC PSychiatry 2019; 19: 44.

10 Paffard M. Suicidal ideation. In Acute Medicine: A Symptom-Based Approach (eds S Haydock, D Whitehead, Z Fritz): 415-20. Cambridge University Press, 2014.

11 Nock MK, Green JG, Hwang I, MCLaughlin KA, Sampson NA, Zaslavsky AM, et al. Prevalence, correlates, and treatment of lifetime suicidal behavior among adolescents: results from the national comorbidity survey replication adolescent supplement. JAMA Psychiatry 2013; 70(3): 300-10.

12 Dazzi T, Gribble R, Wessely S, Fear NT. Does asking about suicide and related behaviours induce suicidal ideation? What is the evidence?. Psychol Med 2014; 44: 3361-3.

13 Mishara BL. Conceptions of death and suicide in children ages 6-12 and their implications for suicide prevention. Suicide Life Threat Behav 1999; 29(2): 105-18.

14 Ballard ED, Tingey L, Lee A, Suttle R, Barlow A, Cwik M. Emergency department utilization among American Indian adolescents who made a suicide attempt: a screening opportunity. J Adolesc Health 2014; 54(3): 357-9.

15 National Confidential Enquiry into Suicide and Safety in Mental Health (NCISH). Suicide by Children and Young People. NCISH, 2017 (https://sites.manchester. ac.uk/ncish/reports/suicide-by-children-and-young-people/)

16 National Institute for Health and Care Excellence (NICE). Treatment Occurring in Paediatrics (STOP) - Assessing and Monitoring Risk of Suicide in Children and Adolescents. NICE, 2014 (https://www.nice.org.uk/sharedlearning/treatmentoccurring-in-paediatrics-stop-assessing-and-monitoring-risk-of-suicide-in-children-and-adolescents).

17 Horowitz L, Tipton Mv, Pao M. Primary and secondary prevention of youth suicide. Pediatrics 2020; 145(2): S195-203.

18 Beckman K, Mittendorfer-Rutz E, Waern M, Larsson H, Runeson B, Dahlin M. Method of self-harm in adolescents and young adults and risk of subsequent suicide. J Child Psychol Psychiatry 2018; 59(9): 948-56.

19 Hawton K, Bale L, Brand F, Townsend E, Ness J, Waters K, et al. Mortality in children and adolescents following presentation to hospital after non-fatal selfharm in the multicentre study of self-harm: a prospective observational cohort study. Lancet Child Adolesc Health 2020; 4(2): 111-20.

20 Bilsen J. Suicide and youth: risk factors. Front Psychiatry 2018; 9: 540

21 Posner K, Melvin GA, Stanley B, Oquendo MA, Gould M. Factors in the assessment of suicidality in youth. CNS Spect 2007; 12: 156-62.

22 Orri M, Scardera S, Perret LC, Bolanis D, Temcheff C, Séguin JR, et al. Mental health problems and risk of suicidal ideation and attempts in adolescents. Pediatrics 2020; 146(1): e20193823. 
23 Orri M, Galera C, Turecki G, Forte A, Renaud J, Boivin M, et al. Association of childhood irritability and depressive/anxious mood profiles with adolescent suicidal ideation and attempts. JAMA Psychiatry 2018; 75(5): 465-73.

24 Storch EA, Sulkowski ML, Nadeau J, Lewin AB, Arnold EB, Mutch PJ, et al. The phenomenology and clinical correlates of suicidal thoughts and behaviors in youth with autism spectrum disorders. J Autism Dev Disord 2013; 43(10): 2450-9.

25 Balazs J, Kereszteny A. Attention-deficit/hyperactivity disorder and suicide: a systematic review. World J Psychiatry 2017; 7(1): 44.

26 Ballard ED, Bosk A, Snyder D, Pao M, Bridge JA, Wharff EA, et al. Patients' opinions about suicide screening in a pediatric emergency department. Pediatr Emerg Care 2012; 28(1): 34-8.

27 Horowitz L, Ballard E, Teach SJ, Bosk A, Rosenstein DL, Joshi P, et al. Feasibility of screening patients with nonpsychiatric complaints for suicide risk in a pediatric emergency department: a good time to talk? Pediatr Emerg care 2010; 26 (11): 787-92.

28 Iannucci J, Nierenberg B. Suicide and suicidality in children and adolescents with chronic illness: a systematic review. Aggress Violent Behav [Epub ahead of print] 14 Feb 2021. Available from: https://doi.org/10.1016/j.avb.2021. 101581.

29 Department of Health. Module 9: Working with Young People on AOD Issues: Facilitator's Guide. 6.1 Brief Interventions - A Definition. Australian Government 2004 (https://www1.health.gov.au/internet/publications/publishing.nsf/Content/ drugtreat-pubs-front9-fa-toc drugtreat-pubs-front9-fa-secb drugtreat-pubs-front9fa-secb-6 drugtreat-pubs-front9-fa-secb-6-1).

30 Cox G, Hetrick S. Psychosocial interventions for self-harm, suicidal ideation and suicide attempt in children and young people: what? how? who? and where? Evid Based Ment Health 2017; 20(2): 35-40.

31 Chand SP, Kuckel DP, Huecker MR. Cognitive behavior therapy. StatPearls Publishing, 2021.

32 Hoagwood KE. Family-based services in children's mental health: a research review and synthesis. J Child Psychol Psychiatry 2005; 46(7): 690-713.

33 Resnicow K, McMaster F. Motivational interviewing: moving from why to how with autonomy support. Int J Behav Nutr Phys Act 2012; 9: 19.

34 World Health Organization (WHO). Effectiveness of Brief Intervention and Contact for Suicide Attempters: a Randomized Controlled Trial in Five Countries. WHO, 2011 (https://www.who.int/bulletin/volumes/86/9/07-046995.pdf).

35 Newton A, Hamm M, Bethell J, Rhodes A, Bryan C, Tjosvold L, et al. Pediatric suicide-related presentations: a systematic review of mental health care in the emergency department. Ann Emerg Med 2010; 56(6): 649-59.

36 Robinson J, Bailey E, Witt K, Stefanac N, Milner A, Currier D, et al. What works in youth suicide prevention? A systematic review and meta-analysis. EClinicalMedicine 2018; 4-5: 52-91.

37 Saunders KE, Smith KA. Interventions to prevent self-harm: what does the evidence say? Evid Based Ment Health 2016; 19(3): 69-72.

38 Anderson N, Ozakinci G. Effectiveness of psychological interventions to improve quality of life in people with long-term conditions: rapid systematic review of randomised controlled trials. BMC PSychol 2018; 6: 11.

39 Pilling S, Fonagy $\mathrm{P}$, Allison $\mathrm{E}$, Barnett $\mathrm{P}$, Campbell $\mathrm{C}$, Constantinou $\mathrm{M}$, et al. Long-term outcomes of psychological interventions on children and young people's mental health: a systematic review and meta-analysis. PLoS One 2020; 15(11): e0236525.

40 Hariton E, Locascio JJ. Randomised controlled trials - the gold standard for effectiveness research: study design: randomised controlled trials. BJOG 2018; 125: 1716.

41 Charrois TL. Systematic reviews: what do you need to know to get started? Can J Hosp Pharm 2015; 68(2): 144.

42 Garritty C, Gartlehner G, Kamel C, King VJ, Nussbaumer-Streit B, Stevens A, et al. Cochrane Rapid Reviews. Interim Guidance from the Cochrane Rapid Reviews Methods Group. Cochrane Rapid Reviews, 2020 (http://methods. cochrane.org/sites/methods.cochrane.org.rapidreviews/files/uploads/cochrane_rr_-_guidance-23mar2020-final.pdf).

43 Tricco AC, Antony J, Zarin W, Strifler L, Ghassemi M, Ivory J, et al. A scoping review of rapid review methods. BMC Med 2015; 13: 224.

44 Moher D, Liberati A, Tetzlaff J, Altman DG. Preferred reporting items for systematic reviews and meta-analyses: the PRISMA statement. BMJ 2009; 339: 332-6.

45 Popay J, Roberts H, Sowden A, Petticrew M, Arai L, Rodgers M, et al. Guidance on the Conduct of Narrative Synthesis in Systematic Reviews. A Product from the ESRC Methods Programme. Lancaster University, 2006 (https://www.lancaster.ac.uk/media/lancaster-university/content-assets/documents/fhm/dhr/ chir/NSsynthesisguidanceVersion1-April2006.pdf).

46 Campbell M, McKenzie JE, Sowden A, Katikireddi SV, Brennan SE, Ellis S, et al. Synthesis without meta-analysis (SWiM) in systematic reviews: reporting guideline. BMJ 2020: 368: 16890
47 Higgins JPT, Savović J, Page MJ, Elbers RG, Sterne JAC. Chapter 8: assessing risk of bias in a randomized trial. In Cochrane Handbook for Systematic Reviews of Interventions version 6.2 (eds Higgins JPT, Thomas J, Chandler J, Cumpston M, Li T, Page MJ, et al.). Cochrane Training, 2021.

48 Wharff EA, Ginnis KB, Ross AM, White EM, White MT, Forbes PW. Family-based crisis intervention with suicidal adolescents: a randomized clinical trial. Pediatr Emerg Care 2017.

49 Diamond GS, Wintersteen MB, Brown GK, Diamond GM, Gallop R, Shelef K, et al. Attachment-based family therapy for adolescents with suicidal ideation: a randomized controlled trial. J Am Acad Child Adolesc Psychiatry 2010; 49 (2): 122-31.

50 Hughes JL, Asarnow JR. Enhanced mental health interventions in the emergency department: suicide and suicide attempt prevention in the ED. Clin Pediatr Emerg Med 2013; 14(1): 28-34.

51 King CA, Gipson PY, Horwitz AG, Opperman KJ. Teen options for change: an intervention for young emergency patients who screen positive for suicide risk. Psychiatr Serv 2015; 66(1): 97-100.

52 Grupp-Phelan J, Stevens J, Boyd S, Cohen DM, Ammerman RT, Liddy-Hicks S, et al. Effect of a motivational interviewing-based intervention on initiation of mental health treatment and mental health after an emergency department visit among suicidal adolescents: a randomized clinical trial. JAMA Netw Open 2019; 2(12): e1917941.

53 Asarnow JR, Baraff L, Berk M, Grob C, Devich-Navarro M, et al. Effects of an emergency department mental health intervention for linking pediatric suicidal patients to follow-up mental health treatment: a randomized controlled trial. Psychiatr Serv 2011; 62(11): 1303.

54 Asarnow JR, Berk MS, Baraff LJ. Family intervention for suicide prevention: a specialized emergency department intervention for suicidal youths. Prof Psychol Res Pract 2009; 40(2): 118-25.

55 Koren $\mathrm{PE}$, DeChillo N, Friesen BJ. Measuring empowerment in families whose children have emotional disabilities: A brief questionnaire. Rehabil Psychol 1992; 37(4): 305-21.

56 Oppenheimer CW, Stone LB, Hankin BL. The influence of family factors on time to suicidal ideation onsets during the adolescent developmental period. J Psychiatr Res 2018; 104: 72-7.

57 Fontanella CA, Warner LA, Steelesmith DL, Brock G, Bridge JA, Campo JV. Association of timely outpatient mental health services for youths after psychiatric hospitalization with risk of death by suicide. JAMA Netw Open 2020; 3(8): e2012887.

58 Barbe RP, Bridge J, Birmaher B, Kolko D, Brent DA. Suicidality and its relationship to treatment outcome in depressed adolescents. Suicide Life Threat Behav 2004; 34(1): 44-55.

59 Negron R, Piacentini J, Graae F, Davies M, Shaffer D. Microanalysis of adolescent suicide attempters and ideators during the acute suicidal episode. J Am Acad Child Adolesc Psychiatry 1997; 36(11): 1512-9.

60 MacDonald S, Sampson C, Turley R, Biddle L, Ring N, Begley R, et al. Patients experiences of emergency hospital care following self-harm: systematic review and thematic synthesis of qualitative research. Qual Health Res 2020; 30(3): 471-85.

61 National Institutes for Health and Care Excellence (NICE). Depression in Children and Young People: Identification and Management. NICE Guideline [NG134]. NICE, 2019 (https://www.nice.org.uk/guidance/ng134).

62 Huang $\mathrm{HC}-\mathrm{H}$, Ougrin D. Impact of the COVID-19 pandemic on Child and Adolescent Mental Health Services. BJPsych Open 2021; 7(5): e145.

63 Rice S, Robinson J, Bendall S, Hetrick S, Cox G, Bailey E, et al. Online and social media suicide prevention interventions for young people: a focus on implementation and moderation. J Can Acad Child Adolesc Psychiatry 2016; 25(2): 80.

64 Jacobs R, Chalkley M, Aragón MJ, Böhnke JR, Clark M, Moran V. Funding approaches for mental health services: is there still a role for clustering? BJPsych Adv 2018; 24(6): 412-21.

65 Clarke D, Usick R, Sanderson A, Giles-Smith L, Baker J. Emergency department staff attitudes towards mental health consumers: a literature review and thematic content analysis. Int J Ment Health Nurs 2014; 23(3): 273-84.

66 Soole R, Kõlves K, de Leo D. Suicide in children: a systematic review. Arch Suicide Res 2015; 19(3): 285-304.

67 Ogundele MO. Behavioural and emotional disorders in childhood: a brief overview for paediatricians. World J Clin Pediatr 2018; 7(1): 9-26.

68 Betz ME, Boudreaux ED. Managing suicidal patients in the emergency department. Ann Emerg Med 2016; 67(2): 276 